\title{
La légende de la ville
}

\section{L'espace urbain dans la culture francophone}

\author{
issue de l'immigration
}

\author{
ERNSTPETER RUHE (WÜRZBURG)
}

C’est la Seconde Génération

Celle des sphynx du bitume

Celle des sphynx du béton. (Mounsi) ${ }^{1}$

„L'écrit est comme une ville à laquelle les mots ouvrent mille portes.“2 ${ }^{2}$ Walter Benjamin flâne du texte à la texture de la ville. Les portes évoquent une clôture à l'ancienne, mais leur extrême multiplication abolit toute enceinte, elle n'en laisse subsister que des débris. Le texte aux mille pores, la ville aux mille portes - deux images se chevauchent et se heurtent, signe avant-coureur de l'éclatement qui menace. Mille portes ne ferment plus rien, elles sont la négation même de la notion de porte. Benjamin lisait la ville à travers Baudelaire et sa notion de la modernité, moment de tension entre passé et présent, d’un côté „l'éternel et l'immuable“ et de l'autre „le transitoire, le fugitif, le contingent". 3

Comparer texte et ville au niveau de l'écriture implique leur ressemblance au niveau de la lecture. Le symbole de cette lisibilité qui permet de dominer le paysage urbain est le regard panoramique. „Les deux mains au

\footnotetext{
${ }^{1}$ Poème-rap de Mounsi, intitulé „Seconde génération“, mis en exergue de son livre Territoire d'outre-ville (Paris: Stock, 1995), 11-12.

„2Geschriebenes ist wie eine Stadt, zu der die Worte tausend Tore sind.“ Walter Benjamin, Betrachtungen und Notizen 1929-1930; cité d'après Willi Bolle, Physiognomik der modernen Metropole. Geschichtsdarstellung bei Walter Benjamin (Köln, Weimar, Wien: Böhlau, 1994) 264.

${ }^{3}$ „La modernité“, ed. Claude Pichois, Baudelaire, Oeuvres complètes II (Paris: Gallimard, Bibliothèque de la Pléiade, 1976) 694-697; 695.
} 
menton, du haut de ma mansarde“, la vue du poète romantique porte loin, ${ }^{4}$ montant des ateliers industriels vers les „clochers, ces mâts de la cité ... qui font rêver d'éternité“. ${ }^{5}$ Mais ce côtoiement du nouveau et de l'ancien qui semble paisible au regard méditatif n'est qu'une étape dans une perpétuelle transition: „... la forme d'une ville / Change plus vite, hélas! que le coeur d'un mortel“. 6

Le pressentiment du poète ne s'est pas trompé. Les métropoles d'aujourd'hui fuient de toutes parts, balayant avec leurs banlieues, leurs citésdortoirs, leurs centres commerciaux et leurs autres zones toute notion de borne. Quiconque rêve encore de vue d'ensemble doit monter très haut et n'aura toujours pas prise sur le tout. Michel de Certeau a vécu au $110^{\mathrm{e}}$ étage du World Trade Center „l'extase de lire le cosmos“ de Manhattan, fasciné et distant à la fois, comme l'affiche ironique à côté de lui: „It’s hard to be down when you're up“. ${ }^{7}$ L'anthropologue sait que pour comprendre il faut descendre, échanger l'abstraction d'un plan contre le chaos du labyrinthe. On ne peut lire la ville qu'au niveau auquel elle s’écrit.

On pourrait s'adresser aux auteurs attitrés qui lancent leurs héros chercheurs de sens dans les métropoles et les voient rentrer bredouilles, de Michel Butor, en passant par Alain Robbe-Grillet jusqu'à Paul Auster, pour ne citer

\footnotetext{
${ }^{4} \mathrm{Cf}$. le même thème p. ex. dans „Elévation“ d’Alfred de Vigny, qui, monté à Montmartre contemple Paris „de haut“ et réfléchit sur l'“immense cité“ (ed. Jacques-Philipe SaintGérand, Alfred de Vigny, Oeuvres complètes. Paris: Garnier-Flammarion, 1978, 362) et chez Chateaubriand (cf. les citations chez Marc Augé, Non-lieux. Introduction à une anthropologie de la surmodernité. Paris: Seuil, 1992, 112 sqq.). Cf. aussi la partie intitulée „Paris à vol d'oiseau“ dans Notre-Dame de Paris de Victor Hugo (ed. Léon Cellier, Paris: GF-Flammarion 1967, 138-160) ou le poème „Soleils couchants“, v. II 13 sqq. dans Les feuilles d'automne du meme poète (Victor Hugo, Oeuvres Poétiques, ed. Pierre Albouy, Paris: Bibliothèque de la Pléiade, 1964, 786-787) ainsi que le fameux défi que lance Eugène de Rastignac du haut du Père-Lachaise à la ville de Paris: „A nous deux maintenant!“ (Le père Goriot, ed. .Pierre-Georges Castex, Balzac, La comédie humaine III. Paris: Bibliothèque de la Pléiade, 1976, 290).

„„Paysage“ vv. 5-8, poème qui ouvre les Tableaux parisiens, ed. Pichois I (1971) 82.

${ }^{6}$ „Le cygne“, vv. 7-8, ed. Pichois [note 2] I 85; cf. v. 29: „Paris change! ...“ Cf. au sujet de ce poème dans lequel „l'on voit se côtoyer, en un voisinage plein d'étrangeté, la neuve industrie et l'antique religion“ les réflexions de Jean Starobinski, „Les cheminées et les clochers“, Magazine littéraire 280, 1990, 26-27.

${ }^{7}$ Michel de Certeau: L'invention du quotidien. t. I Arts de faire (Paris: Union Générale des Editeurs, 1980) 171-172.
} 
que quelques exemples: ${ }^{8}$ toutes les grilles d'organisation, emploi du temps minutieusement contrôlé, cartes détaillées et autres moyens d'enquête s’avèrent inopérants, $\mathrm{M}$. Revel ne révèle rien. La City of Glass oppose ses façades opaques. Un constat s'impose: il en coûte à la littérature dominante de congédier le vieux rêve du regard plongeant, panoptique.

Descendons jusqu'en bas: De Certeau, ethnologue du proche, s'est mis sur les traces de ce qu'il appelle „les pratiquants ordinaires de la ville““ ${ }^{9}$ Nous ferons de même, tout en changeant de position: nous irons là où les pratiquants ne s'inscrivent pas seulement dans le tissu urbain par leurs manières de faire que le spécialiste déchiffrera, mais là où ils s’écrivent, se chantent, se mettent en scène: dans leur littérature, leur musique, leurs films, leurs danses. Le terrain de notre petite enquête se limite à la culture issue de l'immigration en France. Son analyse tentera de montrer que ces praticiens du paysage urbain n’ont pas de ce qu'ils vivent „une connaissance aussi aveugle que dans le corps à corps amoureux“ dont leur échappe „la lisibilité“, comme le veut De Certeau. S’ils savent s’écrire, c’est qu'ils savent aussi se lire.

Les sans-lieux. - Le titre du dernier livre de Mounsi propose un néologisme intéressant: Territoire d'outre-ville. Echo aux Mémoires d'outre-tombe? L’auteur dit qu'il écrit après une vie mouvementée, „une sorte de traversée initiatique du Styx“.${ }^{10}$ A lire le livre qui oscille entre autobiographie et essai, il devient évident que le titre est au-delà de toute invention allusive une innovation faite par pure nécessité. Vue de la banlieue, la ville est dépassée, comme évanouie, absente. Les nombreuses oeuvres d'auteurs issus de l’immigration, romans, films et rap confondus, en sont la preuve.

\footnotetext{
${ }^{8}$ Michel Butor, L'emploi du temps (Paris: Les Editions de Minuit, 1956). - Alain RobbeGrillet, Projet pour une révolution à New York (Paris: Les Editions de Minuit, 1970). - Paul Auster, City of Glass (Los Angeles: Sun \& Moon Press, 1985): dans ce roman, le dessin de plans permet le déchiffrement du 'message’ „Tower of Babel“; cette ville mythique est évoquée souvent par les chanteurs de rap quand il parlent de leur espace urbain, cf. le vers „Babylone m’étreint“ dans la chanson „C’est arrivé près d’chez toi“ du groupe Suprême NTM de Saint-Denis, ou les chansons „Le biz“ („Babylone t’a brisé“) ou „La sédition“ („Jusqu’à ce que Babylone prenne peur") du film Ma 67 va cracher.

${ }^{9}$ Certeau 173.

${ }^{10}$ Mounsi 41.
} 
Quand elles désignent le cadre urbain, le regard du protagoniste n’englobe que du fragmentaire: „la cité“, „le quartier“, „la zone“, „les tours“, „les blocks“11 - effervescence de notions spatiales qui dit l'évanescence d’une tradition de l'habitat. La Ville, si allusion y est faite, est reléguée dans le mythe qui s'incarne dans les constructions qui ont été érigées au cours du $\mathrm{XIX}^{\mathrm{e}}$ siècle finissant comme pour conjurer un changement qui, à l'époque, s'accélérait inexorablement: Notre-Dame-de-la-Garde pour Marseille, haut dressée sur le rocher (Karim Dridi, Bye-Bye), et la Tour Eiffel pour Paris, que le héros du film Salut cousin d'Allouache Merzak ira regarder comme une pièce de musée pour payer sa dette de touriste. Ce ne sont que les plus longs mâts qui surnagent encore, symboles d'une ère révolue où le regard totalisant était encore possible.

Ma 6T craque. - L’idée d'un ensemble que véhiculait la notion de Ville n’a plus cours. Le temps a passé outre, comme le constate le néologisme de Mounsi, il a nivelé en multipliant. Les lourdes tours des banlieues sont épointées, tronquées. En les réunissant, l’urbanisme a inventé „les ensembles“ et les a affublés de titres traditionnels comme „quartier“ et „cité“, il les a souvent fleuris de noms cache-laideur (Cité des Marguerites, Cité des Pâquerettes, ${ }^{12}$ Cité des Fleurs, ${ }^{13}$ etc.). Sans pitié, la culture des immigrés dénonce le mensonge des termes: en les retournant grâce au verlan, elle les vide de tout sens et leur fait rendre le son disgracieux qui seul leur est approprié: „téci“, „tier-quar“. Ou bien, autre technique de déviation, elle les déforme, en mêlant dans l'écriture chiffres et lettres à l'américaine; au déchiffreur de venir à bout du casse-mot: dans le titre du film de François Richet, il ne reste de la cité qu'un chiffre (6) et une lettre majuscule (T) - Ma $6 T$ va cracker (1997).

Ce qui est ainsi refusé, c'est l'enfermement dans certains mots, parce que ces mots désignent certains lieux. Si cette notion à elle seule déjà „implique

\footnotetext{
${ }^{11}$ Cf. z. B. le titre „Les flammes du mal“ de la bande sonore du film Ma 6T va cracker.

${ }^{12}$ Exemples cités par Mounsi 55.

${ }^{13}$ Mehdi Charef, Le thé au harem d'Archi Ahmed, 22: „La Cité des Fleurs, que ça s'appelle!!! Du béton, des bagnoles en long, en large, en travers, de l'urine et des crottes de chiens. Des bâtiments hauts, longs, sans coeur ni âme. Sans joie ni rires, que des plaintes, que du malheur. Une cité immense entre Colombes, Asnières, Gennevilliers et l'autoroute de Pontoise et les usines et les flics. Le terrain de jeux, minuscule, ils l'ont grillagé! Les fleurs! les fleurs!...“
} 
une indication de stabilité“ ${ }^{14}$, cette stabilité est renforcée du moment que le lieu joue un rôle dans la continuité historique, ce qui, dans les cas de „ville“ et de „cité“ qui nous intéressent ici, élève le lieu au rang de ce que Marc Augé a défini comme un „lieu anthropologique“: ce sont „des lieux de vie produits par une histoire plus ancienne et plus lente“ comme le centre-ville ${ }^{15}$, le lieu de naissance, le lieu des ancêtres, le lieu des monuments et des célébrations annuelles, bref, „l'habitant du lieu anthropologique vit dans l'histoire“ (71) qui l’intègre dans une communauté et lui garantit son identité.

L'architecture des banlieues créée de toutes pièces ne prévoit rien de tout cela. Il est donc logique que la tentative de vouloir fixer leurs habitants dans des lieux par la langue soit dénoncée par l’inversion de la langue, riposte tactique qui subvertit le discours dominant et ses assignations de sens et de résidence. ${ }^{16}$

Marc Augé a opposé au lieu anthropologique le „non-lieu“, produit de ce qu’il appelle „la surmodernité“, dont le signe est „l’excès“, excès d’événements, d'espaces et d'individualisation (42). Ce sont „tous les lieux de hasard et de rencontre où l'on peut éprouver fugitivement la possibilité maintenue de l'aventure ...“ (9), les points de transit tels que les aéroports, les grandes gares, les moyens de transport, les chaînes hôtelières, les parcs de loisir, les grandes surfaces commerciales et les réseaux de communication avec ou sans câble; c'est le monde des consommateurs solitaires. ${ }^{17}$

Ces lieux de passage ne concernent les banlieues qu'en marge. L'activité de leurs habitants s'exerce dans le cadre le plus banal et le plus ouvert: les textes le nomment „la rue“, „le trottoir“, „le macadam“, „le bitume“, „l'asphalte“, „les pavés“18. „Seule issue / la rue“, scande au rythme du rap le

\footnotetext{
${ }^{14}$ De Certeau 208.

${ }^{15}$ Augé 86; pour les autres phénomènes cités cf. 69-78.

${ }^{16} \mathrm{Cf}$. l'étude approfondie du verlan par Adrian V. Fielder, „The Tactical Poetics of Urban Nomadism. A Case Study of Verlan and La haine“, Ernstpeter Ruhe (ed.), Die Kinder der Immigration - Les enfants de l'immigration (Würzburg, Königshausen \& Neumann, 1999) 95-114 (Studien zur Literatur und Geschichte des Maghreb, t. 4).

${ }^{17}$ Augé 101 sqq.

${ }^{18}$ Ces termes reviennent souvent dans les textes de rap, cf. p. ex. l'album avec la trame sonore du film Ma 6-T va cracker, surtout le titre „Les flammes du mal“ (Editions Crépuscule; http://www.multimania.com/hhattack/lyricspouvoir.6t.htm). C’est à juste titre que Mounsi constate: ,„.. les fils de l'immigration sont inscrits dans le bitume ...“ (90). - Je dois à ma fille Cornelia d'avoir eu connaissance de ces textes de rap.
} 
groupe Suprême NTM de Saint-Denis. ${ }^{19}$ Les héros sont dehors et n’arrêtent pas de se déplacer sur les voies publiques et les voies de communication intérieures des maisons, dans les couloirs et les cages d'escalier, zigzaguant au hasard des rencontres et se frayant au besoin de nouveaux chemins sur les toits (Ahmed Bouchaala, Krim, 1995). La mobilité de ceux qui vivent „en osmose avec la rue“ ${ }^{20}$ est totale. Les haltes dans des appartements ou dans des caches de fortune ne marquent que de brèves pauses. Le mouvement de protestation des années 80 est arrivé à son point culminant avec, action spectaculaire, la „Marche des Beurs“ à travers la France. On ne pouvait trouver meilleur symbole pour se définir.

Lieu ou non-lieu, il y a plutôt autre chose. Pour localiser cette mobilisation constante dans la terminologie, il faut un maximum d'ouverture comme seule le garantit la notion d'espace, telle qu'elle est comprise par De Certeau:

L'espace est un croisement de mobiles. Il est en quelque sorte animé par l'ensemble des mouvements qui s'y déploient. ... L'espace serait au lieu ce que devient le mot quand il est parlé, c'est-à-dire quand il est saisi dans l'ambiguïté d'une effectuation ... A la différence du lieu, il n’a donc ni l’univocité ni la stabilité d’un „propre“. (208)

Définition heureuse qui nous permet d'y faire entrer des phénomènes frappants de la culture issue de l'immigration. Ce que le verlan fait subir à la langue, la marche le fait subir à l'espace: en se servant de l'espace public de façon incongrue - transposer au dehors une partie de la vie du dedans, créer des parcours imprévus -, elle déstabilise la distribution des fonctions et y introduit l'ambiguïté. ${ }^{21}$

Le sens de la marche. - Tout est imprégné par le rythme des pas. Il donne aussi le la à la créativité artistique. Phrases courtes et fréquence de dialogues

\footnotetext{
${ }^{19}$ Titre „Laisse pas traîner ton fils“, CD sans titre (Kodak TX 6043).

${ }^{20} \mathrm{Cf}$. le rap intitulé „Le biz“ de la trame sonore citée dans la note précédente: „J’ai appris à vivre en osmose avec la rue.“

${ }^{21}$ De Certeau, fin lecteur de textes allemands (cf. son interprétation d'un poème de Morgenstern, 223), introduit dans sa brève évocation des marcheurs qui écrivent le 'texte urbain ... sans pouvoir le lire“ (174) le terme de „Wandersmänner“, sans le commenter. On voit mal en quoi ce mot plutôt archaïque peut servir l'analyse de pratiques quotidiennes d'aujourd'hui.
} 
aux répliques brèves dans les récits; „calligraphies heurtées“22 des signatures graffitées en coup de poing sur les murs, frappant sec comme le monosyllabe qui les désigne - tag; puis le rap, balancement psalmodique au rythme rapide, ponctué de rimes d’une étonnante richesse: les „poètes des rues“ et „des temps modernes / contre le système qui nous berne“ ${ }^{23}$, comme ils se désignent eux-mêmes, ont déjà bien progressé dans l’invention de ce que Mounsi appelle de ses voeux:

une langue neuve, écrite avec des mots pour ainsi dire sténographiques, rythmiques, extrêmement rapides et en même temps extrêmement expressifs. ${ }^{24}$

L'expressivité va dans la musique et la danse au plus pressé: un code à vé comme Violence traverse les mouvements des corps et des âmes:

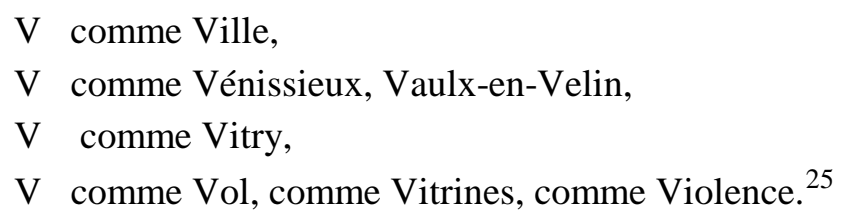

Derrière ce V se dessine en filigrane un V comme victoire: exprimer la violence subie est un premier ,acte de résistance capable d'affirmer“ l'identité26, de combler le vide que l'existence périphérique a creusé. La création permet de passer outre et de gagner „un substitut à une terre“, le „Territoire d’OutreVille“.

Espaces de rêves. - „... tout le malheur des hommes vient d'une seule chose, qui est de ne savoir pas demeurer en repos, dans une chambre.“27 La pensée de Pascal formule la sagesse des sédentaires, qui vivent tranquillement ancrés en lieu sûr.

\footnotetext{
${ }^{22}$ L'expression est de Mounsi 109.

${ }^{23}$ Citations tirées du titre „C’est donc ça nos vies?“ de la trame sonore du film Ma 6-T va cracker (cf. note 14) et du titre „La conscience“ de Matew Star (cf le site http://www.multimania.com/hhattack/lyricsconscience.htm).

2479-80.

${ }^{25}$ Mounsi 20.

${ }^{26}$ Mounsi 42.

${ }^{27}$ Blaise Pascal, Pensées 205 [139], ed. Jacques Chevalier, Pascal, Oeuvres Complètes (Paris: Bibliothèque de la Pléiade, 1954) 1138-1139.
} 
Si la créativité mobile des sans-lieu voit sa récompense dans un „substitut“ territorial, on sent le provisoire, l'inassouvi. Et, en effet, l'analyse des textes des chants et des romans et celle des images de films le révèle clairement: les rêves vont également vers la stabilité d'un lieu anthropologique, à un détail près pourtant qui fait toute la différence: ce n'est pas le lieu fixe où se recueillir en solitaire qui est recherché, mais celui où l'on est bien parce que l'on y est ensemble. Les scènes qui expriment ce désir sont multiples, on peut les grouper en trois catégories, dont les deux dernières sont la conséquence d'une première déception.

La recherche d'un lieu où se retirer afin d'être pour soi à plusieurs est centrée sur ce qui est le plus proche, le bloc de l'immeuble. La superposition de cellules habitables appelées appartements - lieu qui met „à part“, qui isole les familles les unes des autres dans l'anonymat - obéit à des règles de pure rationalisation. L'espoir de pouvoir trouver un lieu de retraite où se réunir, loin de la surveillance familiale, avec les amis du même âge, est vain: même les zones marginales dans les blocs, lieux traditionnels de stockage et de rangement, appartiennent à quelqu'un qui en délogera les occupants-squatters tôt ou tard. C'est le cas des caves, comme le montre de façon drastique le roman Le thé au harem d'Archi Ahmed de Mehdi Charef (1983) et le film qui en a été tiré (Le thé au harem d'Archimède, 1985). C'est le cas aussi des toits auxquels l'urbanisme des banlieues a enlevé le grenier, l'ancienne cache idéale; la rupture avec une longue tradition architecturale se reflète dans la plateforme, qui laisse le plafond du dernier étage à nu; les jeunes essayent malgré tout de s'installer là à l'air libre, y amènent même des meubles rembourrés, mais la position exposée rend inévitables la découverte du 'salon' et l'intervention des tenants de l'ordre (Mathieu Kassovitz, La haine, 1995).

Il est d'ailleurs intéressant de constater que personne ne grimpe sur le toit pour pouvoir jeter un regard panoramique (il ne découvrirait, il est vrai, qu'un horizon fait d'autres tours décapitées), le regard va plutôt vers les autres à l'intérieur du groupe d'amis qui est réuni. La communication devient du même coup radicalement différente: l'échange habituel de courtes répliques rapides comme il se pratique dans la rue se transforme sur le toit en confession, même si cette pause intime ne dure que le temps d'une cigarette (Karim Dridi, Bye bye). En rusant, les usagers de l'architecture des banlieues réussissent à vivre de brefs moments stabilisants dans le seul espace non utilisé. 
Le lieu anthropologique reste hors d'atteinte dans les cités. Il est relégué dans le rêve nostalgique qui se nourrit des expériences collectives: l'utopie prend modèle sur le passé de la culture d'origine.

La première génération des immigrés était encore parfois arrivée à sauvegarder les traditions de la communauté, ne serait-ce que pour un certain temps: le petit bidonville du Chaâba, dans lequel grandit le gone Azouz Begag en marge de Lyon, reconstitue autour du père le village clanique de Kabylie, ses structures, ses coutumes, ses valeurs, sa pauvreté aussi. Cette idylle de la vie à l'ancienne est trop complète pour ne pas être vouée complètement à l'échec dans le contexte de l'autre culture que l'on essaie d'ignorer. L’observation de règles prescrites par la religion (égorger en privé comme dans le bled) est une infraction aux normes hygiéniques de la société française qui fait intervenir les forces de l'ordre, ce qui ébranle une des assises de la communauté kabyle, l’honneur du chef. Et le coup porté par la seconde génération n’est pas moins décisif: la bicyclette, cadeau offert lors de la fête de la circoncision, est le symbole même de la mobilité des jeunes qui ne vont pas tarder à échapper à l'emprise de l'autorité paternelle; écraser les vélos à coup de masse parce que les enfants ont transgressé les prescriptions restrictives du chef n'est que l'expression de la violence du désespoir: la culture immigrée va devoir s’ouvrir. Le lieu recréé à l'ancienne éclate, ses habitants sont projetés dans l'espace des cités. ${ }^{28}$

C’est l'expérience de ce nouveau monde de la mobilité qui renforce le désir du lieu fixe. Où s’enraciner? Les réponses de la seconde génération qui grandit dans les tours des banlieues trouvent leur dénominateur commun dans l'invention d'un entre-deux. Les images pour meubler cet espace utopique peuvent rester de purs symboles, comme c'est le cas pour le héros du roman Les A.N.I. du 'Tassili' d'Akli Tadjer, qui rêve de pouvoir s'asseoir dans un „immense transat qui, une fois déplié, s’étale de Tamanrasset à Dunkerque“ ${ }^{29}$, ou pour Sakinna Boukhedenna dans son Journal 'Nationalité: im-

\footnotetext{
${ }^{28}$ Azouz Begag, Le gone du Chaâba (Paris: Les Editions du Seuil, 1986), 113-117 et 122 sqq.

${ }^{29}$ Paris: Les Editions du Seuil, 1980, 174.
} 
migré(e)’ qui rêve „de construire une île entre Marseille et Alger, pour enfin qu’on ait, nous, les immigrées et immigrés, la paix.“30

Leïla Sebbar ancre son symbolisme plus concrètement dans la terre: dans le troisième volume de la trilogie composée autour de Shérazade, la fille d'immigrés, un vieil olivier est transplanté du Sud dans une banlieue parisienne. Ce qui ne devait être à l'origine qu'un simple décor pour le temps de la mise en scène d'un film, est immédiatement adopté par les habitants et décoré „,comme font les femmes de l'autre rive à l'arbre qui jouxte le marabout du saint qu'elles vont, ensemble, consulter“31. Le vieil arbre prend racine et commence même à porter des fruits. S'il est porteur aussi d'un symbolisme évident (les deux générations de l'immigration), il incarne surtout aussi le rêve de pouvoir faire se joindre deux cultures, en intégrant la culture d'origine, attachée au sol, à la terre d'un monde fait de béton.

L’optimisme qui s'exprime dans ce projet n'est plus partagé par les auteurs et cinéastes de la génération suivante, les constructions de lieux ouvertement utopiques d'Akli Tadjer et de Sakinna Boukhedenna le montraient déjà. Le film Bye bye de Karim Dridi (1995) traduit dans la scène finale le même mélange ambigu de désirs et de distanciation. Les deux frères qui ne veulent ni vivre dans le monde où le père immigré impose à ses enfants son rêve du retour dans le village d'origine, ni risquer de s'enliser dans les tentations du ghetto d'immigrés à Marseille, cherchent où aller. Le regard panoramique plonge de la côte vers le Sud, sur la mer, un des leitmotivs du film: „Allons en Espagne!“ propose plein d'entrain le jeune frère, et sur la bande sonore retentissent au même moment les notes engageantes d'un flamenco. Confirmation d'un bon choix qui garantit au film une fin optimiste? La musique est plutôt un commentaire ironique au rêve que font nos chercheurs de terre: le paradisiaque El-Andalous, depuis la chute de Grenade lieu de tous les désirs pour le monde islamique, a pour seule réalité le lieu commun. Vouloir rentrer en Espagne serait courir après les fantômes d'un souvenir collectif.

\footnotetext{
${ }^{30}$ Paris: L’Harmattan, 1987, 103. Cf. à ce sujet aussi Cornelia Ruhe, „Die Mythologie der croisés. Kultur und Identität in der Shérazade-Trilogie von Leïla Sebbar“, Romanische Forschungen 2000 (sous presse).

${ }^{31}$ Leïla Sebbar, Le fou de Shérazade (Paris: Stock, 1991), 197.
} 
Les strates de l'histoire: Straßburg. - Comment dépasser la mélancholie de l'utopie et imaginer un lieu anthropologique concret pour les immigrants? Faut-il, pour pouvoir le faire, avoir plus de distance que ne l'a quelqu'un qui est directement concerné? Dans son dernier roman Les nuits de Strasbourg (1997), Assia Djebar a développé un scénario qu’on peut lire comme une esquisse de réponse.

Le choix du lieu est particulièrement heureux: La ville de Strasbourg porte un nom-symbole qui définit cette ville par l'espace propre à la société issue de l'immigration: die Straße, la rue et la route; le mot désigne en allemand les deux à la fois. La ville sur la frontière est une ville-carrefour où différentes cultures se croisent et se mélangent; les immigrés maghrébins en font partie depuis longtemps. L'espace urbain n'aurait donc rien de particulier, comparé aux autres grandes villes de France. Il faut un regard neuf pour découvrir ce qui est spécifique, il ne faut pas rester à la surface, mais aller en profondeur.

La jeune héroïne du roman, l'Algérienne Thelja - tout comme l'auteur d'ailleurs - est historienne. Strasbourg, de par sa position, est un lieu particulièrement riche d'histoire et de traditions. Thelja fouille intensément le passé de la même manière qu'elle parcourt inlassablement les voies du présent, les rues de Strasbourg. C'est en fouillant dans les archives et les bibliothèques de la ville et en se promenant à travers les strates de l'histoire qu'elle découvre ce qui a été oublié ou évincé par la mémoire collective des Strasbourgeois: tout comme les immigrés, les tenants du lieu ont été colonisés eux aussi, et plusieurs fois même, par les Allemands et les Français; et, point commun encore plus important, ils sont aussi des 'immigrés' dans leur propre ville: face à la menace allemande au début de la Seconde Guerre mondiale, Strasbourg avait été vidée de sa population pour de longs mois.

Tout le monde est donc logé à la même enseigne par l'expérience de la déroute et de l'errance, ce qui devrait garantir la tolérance et l'entente, si précaires soient-elles, entre celles et ceux qui jonglent sur les multiples frontières. Thelja sonde les profondeurs de la société pour que les leçons de l'histoire fassent changer la surface, la vie quotidienne entre les différentes communautés. Et elle va de l'avant jusque dans sa vie intime et surmonte son plus grand tabou en acceptant un Français pour amant. 
La jeune Algérienne parviendra-t-elle à se faire fondre le passé et le présent en une identité commune? Elle ne s'appellerait pas Thelja, c'est-à-dire Neige, si cela était son rôle. C'est elle qui fond. Après neuf nuits d'amour, elle disparaît de la ville, incitant ainsi son amant alsacien, sédentaire, à l’imiter et à „devenir nomade“ (108).

En suspens. - Vivre ainsi dans une continuelle mobilité, c’est transformer profondément la notion de lieu anthropologique. Du moment que les cultures différentes ont tant d'expériences en commun, les différences entre immigrés et sédentaires n'ont plus de raison d'être. Et le lieu anthropologique perd son caractère de lieu unique d'attachement identitaire. La stabilité est désormais dans la permanence de la mobilité. Les lieux, ouverts à tous les métissages, sont interchangeables.

C'est une conception exigeante qui n'est pas facile à vivre. Thelja ellemême en est la preuve. A la fin du roman, elle revient clandestinement à Strasbourg et s'y promène la nuit. Quand elle grimpe à la fin tout en haut de la flèche de la cathédrale, elle choisit pour son ascension le symbole classique du lieu anthropologique traditionnel. Regret nostalgique de la nomade brune qui aurait trop risqué en voulant se faire joindre espace et lieu? C'est plutôt un adieu douloureux à un passé qui la hante encore, mais qui ne peut plus être désormais qu'une citation culturelle. Elle monte la nuit, à l'aube, son regard panoramique ne rencontrera que du vide, un cri s'élèvera: Est-ce le suicide qui menace? Le livre se termine par trois points de suspension, comme reste suspendu le lieu anthropologique des immigrés dans „le transitoire, le fugitif, le contingent“. 


\section{Panorama Bourbaki in Luzern:}

Rundbild in Rundgebäude vom Ende 19. Jh, jetzt restauriert!

General Bourbaki führte Ostarmee Frankreichs im Krieg 1870/71; geschlagene Armee wurde 1871 in der Schweiz interniert - Panorama zeigt Ankunft der Armee

Panorama im 19. Jh. großer Erfolg, in festen Gebäuden oder auch mobil für Jahrmärkte

gestattete dem Zuschauer weit zu sehen und einen Überblick zu haben über historische Ereignisse (cf. Arromanches, Invasions-Museum: Zuschauer im Cockpit eines Bombers, der auf Küsten zufliegt)

+ mitten drin zu sein

am Rand unterhalb von Bild in Zwischenraum zu Zuschauergalerie Übergang mit dreidimensionalen Figuren (Menschen etc.) - Übergang gleitend aus Realität zu Wandbild

cf. barocke Malerei, bei der Beine heraushängen, Residenz Würzburg

Victor Hugo: Soleils couchants II, in Les feuilles d'automne

v. 13 sqq: Oh! qui m'emportera sur quelque tour sublime

d'où la cité sous moi s’ouvre comme un abîme!

v. 30 Je regarde à mes pieds la géante [la vielle cité, v. 25] dormir!

ed. Pléiade 786-786

Babylone:Jusqu’à ce que Babylone prenne peur (Ma 6T, La sédition)

Babylone t’a brisé (Le biz)

Babylone m'étreint (C'est arrivé près d'chez toi, groupe NTM/SaintDenis) 